\title{
Adult pancreatic cavernous hemangioma: case presentation of a benign tumor with a complex composition
}

\author{
Tao Lianyuan, Wang Yafeng, Yu Haibo, Dong Yadong, Ma Jiahao, Lu Yuanxiang and Li Deyu* (D)
}

\begin{abstract}
Background: Pancreatic cavernous hemangioma is an extremely rare benign tumor that is difficult to diagnose on an imaging examination, and its histopathological examination has rarely been reported.

Case presentation: Herein, we present the case of a 63-year-old man who was admitted to the hospital due to left upper abdominal pain and defecation unformed for more than 2 years. None of the positive results obtained from the physical examination could explain his symptoms. The imaging examination indicated a multilocular cyst with septa in the head of the pancreas. The patient underwent a pancreaticoduodenectomy, and the pathologic diagnosis was pancreatic cavernous hemangioma. The histopathological examination showed that the lesion was positive for benign vascular markers, such as CD31, CD34 and F8, and negative for lymphocyte markers, such as D2-40. Moreover, it was also positive for ERG and cytokeratin markers, CAM5.2 and AE1/AE3, indicating the complexity of its components, and Ki-67 negativity revealed its benign nature.

Conclusions: Pancreatic cavernous hemangioma has a complex composition that may be reflected not only in the imaging examination but also in the immunohistochemical detection, and it may achieve a good outcome by surgical excision.
\end{abstract}

Keywords: Cavernous hemangioma, Pancreas, Adult, Immunohistochemical

\section{Background}

As a congenital malformation of the vascular system, cavernous hemangioma is an uncommon type of primary cystic neoplasm. The occurrence of pancreatic hemangiomas in adults is extremely rarely reported [1$21]$, especially that of cavernous hemangioma $[9,15,21]$. Adult pancreatic hemangiomas often manifest as large cystic lesions in middle-aged females, and in many cases, the patients exhibit abdominal pain but no evidence of malignancy $[11,13,16,19]$. The major symptoms of cavernous hemangioma are abdominal pain and distension associated with the enlarged tumor $[1,7,11]$.

The diagnosis of cavernous hemangioma remains controversial $[1,7,11]$. The expression of surface molecular markers is still unclear. In the present study, we analyzed the clinical features and immunohistochemical

\footnotetext{
* Correspondence: lideyu19@hotmail.com

Department of Hepatobiliary Surgery, Henan Provincial People's Hospital,

People's Hospital of Zhengzhou University, School of Clinical Medicine,

Henan University, No. 7 Weiwu Road, Zhengzhou 450003, China
}

data of a rare case of an adult patient with pancreatic cavernous hemangioma without recurrence over 2 years following curative surgery.

\section{Case report}

A 63-year-old man was admitted to the hospital due to left upper abdominal pain and defecation unformed for more than 2 years. There was no history of acute pancreatitis or abdominal trauma. The patient had no prior surgeries. He was on no medications. The family history was noncontributory. The physical examination was normal.

Endoscopic ultrasonography (EUS) showed a mixed echo with a range of $105 \times 82 \mathrm{~mm}$ (mainly a cystic echo with visible separation and a visible range $59 \times 47 \mathrm{~mm}$ heterogeneous hyper echo) within an unclear contour of the head of the pancreas. CDFI indicated a high echo and visible blood flow within the separation. CT revealed a well-defined cystic mass in the head of the pancreas, with the formation of internal calcifications (Fig. 1). 

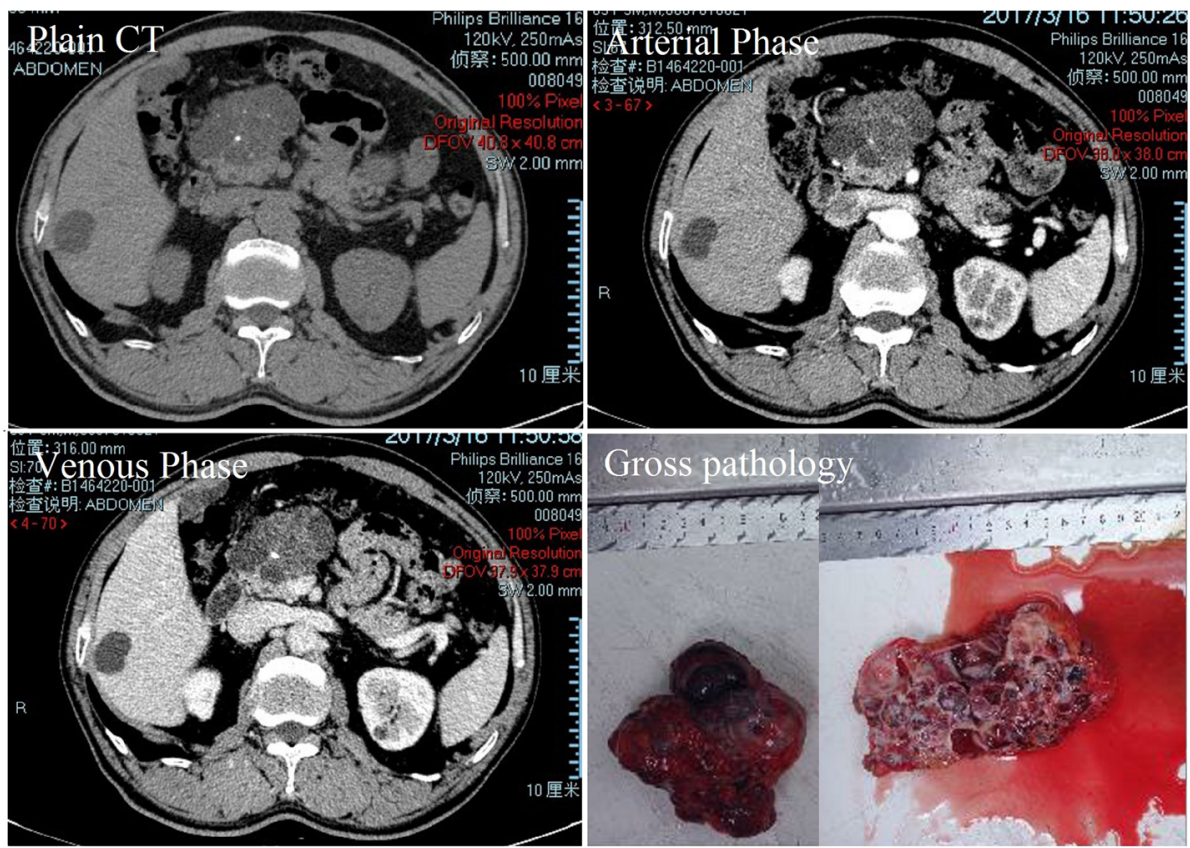

Fig. 1 The tumor was a multilocular cyst with septa and fluid-fluid levels. Enhanced scan showing "fast in, slow out". CT enhancement (arterial phase and venous phase): The CT value was slightly higher than that of plain CT after contrast agent injection at the base of the tumor. Gross pathology showed a cystic lesion with thick septa

Laboratory tests showed that the complete blood count, complete metabolic panel, serum amylase and lipase, coagulation panel, fasting lipid profile, and serum CA 19-9 and CEA levels were normal. The detailed results were as follows: leukocyte count $6.89 \times 10^{9} / \mathrm{L}$, neutrophilic granulocyte count $4.31 \times 10^{9} / \mathrm{L}$, neutrophil percentage $62.5 \%$, lymphocyte count $2.03 \times 10^{9} / \mathrm{L}$, lymphocyte percentage $29.5 \%$, RBC $4.6 \times 10^{12} / \mathrm{L}$, hemoglobin $146 \mathrm{~g} / \mathrm{L}$, platelet $163 \times 10^{9} / \mathrm{L}$, alanine aminotransferase $15 \mu \mathrm{L}$, aspartate aminotransferase, albumin $37.5 \mathrm{~g} / \mathrm{L}$, total bilirubin 9.5 $\mathrm{mol} / \mathrm{L}$, direct bilirubin $2.7 \mu \mathrm{mol} / \mathrm{L}$, alkaline phosphatase $68 \mathrm{U} / \mathrm{L}$, glutamyl transpeptidase $15 \mathrm{U} / \mathrm{L}, \mathrm{HBsAg}(-)$, $\mathrm{HBeAg}(-), \mathrm{HBeAb}(-), \mathrm{HBcAb}(-), \mathrm{HCV}-\mathrm{Ab}(-)$, and CEA $14.01 \mathrm{ng} / \mathrm{mL}$. AFP and CA199 showed no abnormalities.

A pancreaticoduodenectomy was performed, and a pink-brown, multiloculated and approximately $10 \mathrm{~cm} \times$ $5 \mathrm{~cm} \times 5 \mathrm{~cm}$ cystic solid mass with a clear boundary and a firm texture was found at the head of the pancreas (Fig. 1). No evidence of invasion of the portal vein or the superior mesenteric vein or other vessels was found. No bleeding occurred during or after the operation, and the patient had a smooth postoperative recovery.

The microscopic examination indicated a cystic structure extending into the interlobular septa of the pancreatic parenchyma that consisted of dilated vascular structures lined by endothelial cells. Some of the vessels with a membranous wall were filled with serous fluid rather than blood, indicating that those vessels do not participate in the circulation (HE 10x). Some of the vessels in the tumor were well-identifiable arteries with thick walls characterized by profusely proliferating multilayer endothelial cells; other vessels had thin, membranous walls with a single layer of flattened cells (HE 40x), with uniform nuclei and no atypical nuclei (Fig. 2). Immunohistochemical (IHC) staining showed that the lining was positive for CD31, CD34 and F8, focally positive for ERG, and negative for D2-40, ER and Ki-67, supporting the diagnosis of hemangioma (Fig. 2). However, the cytokeratin markers, CAM5.2 and AE1/AE3, were also positive (Fig. 3). All 12 lymph nodes had a normal histology. Postoperatively, the patient tolerated an oral intake of liquids on the third day, was discharged on the 7th postoperative day and remained symptom free 2 years after surgery.

\section{Discussion and conclusions}

Vascular tumors include lymphangiomas, hemangiomas, lymphangiomas, angiotheliomas, angioblastomas, and angiosarcomas, are of which all cystic lesions. As a benign neoplastic lesion with slow growth and rarely malignant changes, these lesions are commonly found in the skin, eyes, liver, brain, spleen and other organs, while cavernous hemangioma occurring in the pancreas is extremely rare [22]. Pancreatic cavernous hemangioma is more common in women than in men (the male to female ratio is 1:3), the exact cause of the disease is unknown, and it is mostly recognized as a congenital disease $[11,15]$. The age of the patients at diagnosis 

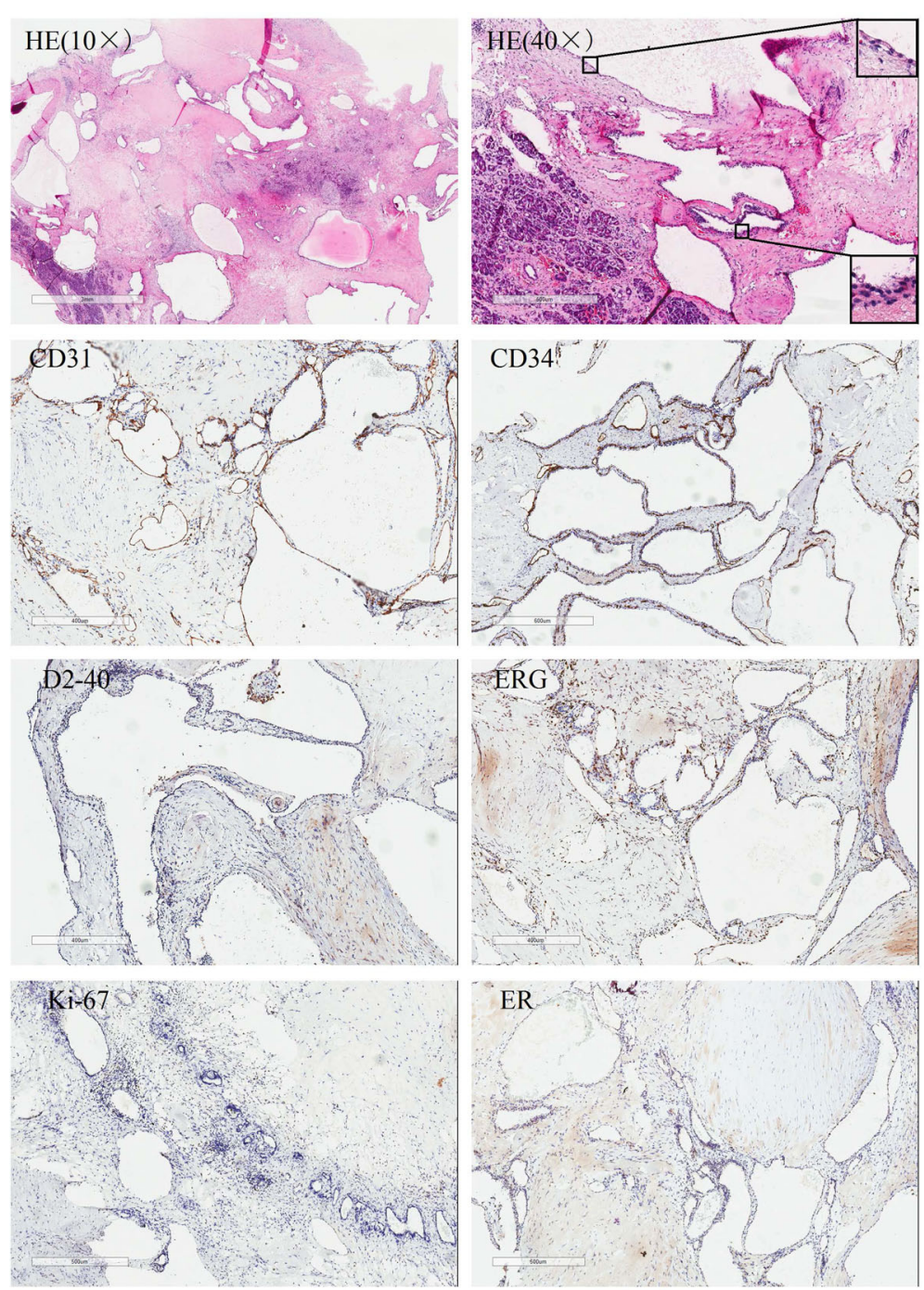

Fig. 2 H\&E staining of the tumor showing a cyst extending into the interlobular septa of the pancreatic parenchyma (HE 10X). Some of the vessels in the tumor were well-identifiable arteries with thick walls characterized by profusely proliferating multilayer endothelial cells; other vessels had thin, membranous walls with a single layer of flattened cells (HE 40X). Immunohistochemical staining indicated positivity for CD31 and CD34, focal positivity for ERG, and negativity for D2-40, ER and Ki-67 (40x)
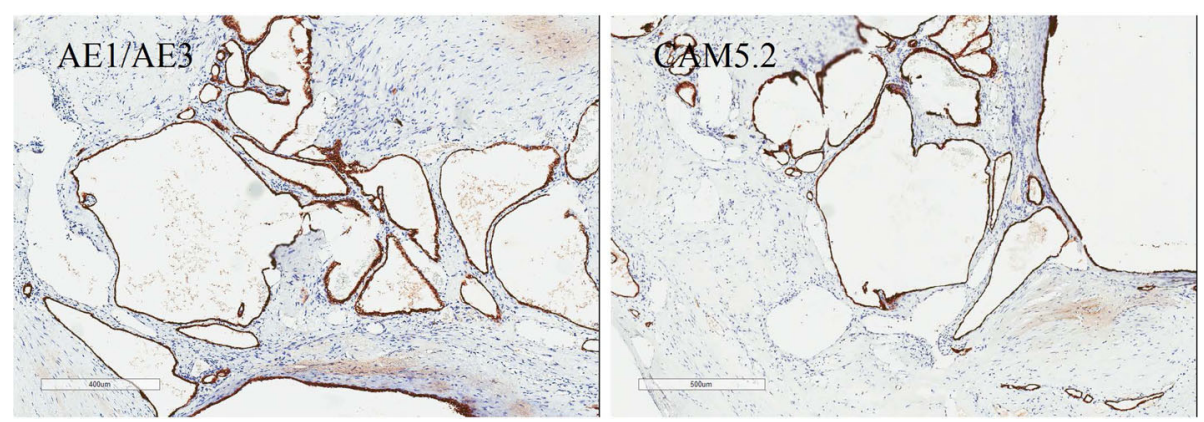

Fig. 3 Immunohistochemical staining showing a cavernous, ectatic endothelial neoplasm positive for CAM5.2 and AE1/AE3(40X) 
ranges from 18 to 79 years, and it occurs more frequently in childhood than adulthood [1-21]. It progresses slowly to the stage of degeneration after hyperplasia in infancy and gradually disappears after many years, leaving traces of fiber and fat in adulthood [11, 15, 23]. Adult pancreatic hemangioma is very rare, and its pathological nature is completely different from that of childhood pancreatic hemangioma $[11,15,23]$.

Cavernous hemangioma of the pancreas shows no typical clinical symptoms, and the majority of patients experience pain and discomfort in the middle and upper abdomen [11, 15]. Nausea, abdominal distention, fever, episodic dizziness and palpitations may be associated with pain. Some patients come to hospital because of low back pain, a palpable abdominal mass or gastrointestinal bleeding [2]. Occasionally, it may be diagnosed from incidental findings on an imaging examination during a physical examination. Neither episodes of pancreatitis nor a family history of pancreatic diseases has been reported before. It may rarely be observed in the setting of von HippelLindau disease, especially in young patients [24].

The imaging manifestations of pancreatic cavernous hemangioma are diverse. Abdominal X-ray and cholangiography are of little significance in the diagnosis of pancreatic cavernous hemangioma, while digital subtraction angiography (DSA) is invasive and rarely used [15]. Pancreatic cavernous hemangioma is usually characterized by ultrasonography as well demarcated and appearing overall hyper- or isoechogenic to the rest of the pancreas with mixed echogenicity or an irregular low echo and usually no blood flow signal or a low-velocity blood flow signal. Contrast-enhanced ultrasound has revealed that cavernous hemangiomas are typically rich in blood supply, but a large portion of pancreatic cavernous hemangiomas do not show obvious enhancement, so the nature of the lesion cannot be determined.

CT and MRI are the main imaging examinations used to diagnose cavernous hemangioma of the pancreas [ 9 , 15, 21]. Typically, hemangiomas can be significantly enhanced in the arterial phase of the CT scan, but pancreatic cavernous hemangioma is a cystic tumor that usually contains neurovascular components and is accompanied by an arteriovenous shunt, and the flow rate is slow when the blood flow passes through, so it may lead to no enhancement in the arterial phase. At the same time, the proportion of cystic and solid components in the tumor is different, which may lead to different degrees of enhancement in the arterial phase [25]. The CT manifestations of the present case were an atrial cystic mass, moderately enhanced in the base of the mass, but the mass itself did not show significant enhancement. Many studies [2, 9, 15, 25] have shown that pancreatic cavernous hemangioma does not necessarily show significant enhancement in the arterial phase of the CT scan, so it is believed that the diagnosis of this disease cannot be ruled out without obvious enhancement in the arterial phase. Moreover, in patients with pancreatic cavernous hemangioma, only a very small number of patients can present characteristic changes, and the vast majority of imaging diagnoses are difficult. Therefore, it may be easy to confuse pancreatic cavernous hemangioma with other pancreatic lesions (such as pseudocysts, serous cystadenomas, mucinous cystadenomas and intraductal papillary mucinous neoplasms, IPMNs) [7]. There was no enhancement of pancreatic pseudocysts on the CT examination. The CT findings of serous cystadenoma and mucinous cystadenoma are similar to those of pancreatic cavernous hemangioma. The margin of a mucinous cystadenoma is smooth, with or without septa, and is usually surrounded by eggshell-like calcifications. IPMN is a pleomorphic cystic mass, and the key of its diagnosis is communication with the main pancreatic duct. In addition, cavernous hemangioma of the pancreas should be differentiated from other diseases of the pancreas that are rich in blood supply, such as neuroendocrine tumors, metastatic renal cell carcinomas, an intrapancreatic accessory spleen and arteriovenous malformation [23]. A comprehensive review of the relevant literature revealed the following points to aid in the diagnosis $[9,15,21], 1)$ no skin or scleral yellow staining; (2) tumor markers were mostly negative; (3) CT or MRI examination revealed mostly a circular mass with a clear boundary and the coexistence of cystic and solid components, no main pancreatic duct dilation, and enhanced scan lesions can be slightly enhanced; (4) ultrasound examination showed a high-echo mass, no blood flow signal or a low-speed blood flow signal; and (5) no lymphatic or distant metastasis. In short, cavernous hemangiomas typically have a characteristic manifestation in CT or MRI of an enhancement feature described as "fast in and slow out", which indicates that edge nodular and patchy enhancement appear at the arterial stage, and the enhancement range spreads to the center at the portal vein stage, which is followed by delayed scanning maintained at an equal or slightly high density.

At present, the gold standard for the diagnosis of pancreatic cavernous hemangioma is still a pathological diagnosis. Microscopically, cavernous hemangioma is mainly composed of a dilated abnormal sinus, lined with monolayer vascular endothelial cells, and the fibrous tissue in the sinus is not completely spaced to form a cavernous structure $[11,15]$. Depending on the size of the vascular spaces, they can be capillary or cavernous. Positivity for CD31 and CD34 indicates hemangioma, lymphangioma and other benign vascular tumors, and negativity for D2-40 and a lack of lymphocytes help to exclude lymphangioma $[9,15,21]$. Focal positivity for ERG and negativity for Ki-67 indicate the complexity of 
the components and the benign nature of the tumor. Our study denies the association between cavernous hemangiomas and increased levels of estrogen or negativity for ER. However, as markers of cytokeratin, which is supposed to be negative in hemangiomas [1], CAM5.2 and AE1/AE3 were positive in the present case, which may also suggest a tendency toward epithelialization and indicate the complexity of tumor components. A precise histopathological diagnosis of hemangioma is of great significance, as other vascular tumors may require more radical surgical margins at resection, adjunctive therapy and closer postresection follow-up. For example, the recurrence rate of hemolymphangioma is as high as 10 to $27 \%$ after complete resection [26].

Surgical excision is still the best treatment because almost all resected pancreas hemangioma cases have good outcomes, with the resolution of symptoms and no tumor recurrence $[9,15,21]$. However, because a preoperative qualitative diagnosis is very difficult, the choice of treatment is also very controversial. By fully understanding the imaging characteristics of pancreatic cavernous hemangioma and combining clinical and relevant laboratory examinations (especially tumor markers), the possibility of cavernous hemangioma can be considered while malignant lesions are excluded to eliminate or reduce the risk of surgery and postoperative complications. Our study presents a case of surgical removal of a giant cavernous hemangioma without recurrence at the 2-year follow up.

In summary, pancreatic cavernous hemangioma is a rare tumor with a complex composition that is reflected not only in the imaging examination but also in the immunohistochemical detection, and it can achieve a good outcome by surgical excision.

\section{Abbreviations}

CDFI: Color doppler flow imaging; CEA: Carcinoembryonic antigen; CT: Computed tomography; DSA: Digital subtraction angiography; EUS: Endoscopic ultrasonography; IHC: Immunohistochemical; MRI: Magnetic resonance imaging

\section{Acknowledgements}

Not applicable.

\section{Authors' contributions}

LD and TL conceived and designed this study; TL, WY, YH, JM, YD, and LY performed the analysis and interpreted the data; TL and LD wrote the manuscript. All authors read and approved the final manuscript.

\section{Funding}

This study was supported in part by research funding granted to TL from the Doctoral Venture Capital Fund of Henan Provincial People's Hospital (No. ZC20180077) and the Special Project of Henan Provincial Key Research, Development and Promotion (Science and Technology) (No. 20190274). The founders had no role in study design, data collection and analysis, decision to publish, or preparation of the manuscript.

\section{Availability of data and materials}

The authors declare that all data supporting the findings of this study are available within the article. No datasets were generated or analyzed during the current study.

Ethics approval and consent to participate

The current study was approved by the Clinical Ethics Committee of Henan Provincial People's Hospital.

\section{Consent for publication}

Written informed consent was obtained from the patient for publication of this study and any accompanying images.

\section{Competing interests}

The authors declare that they have no competing interests.

Received: 17 May 2019 Accepted: 15 November 2019

Published online: 27 November 2019

References

1. Bursics A, Gyokeres T, Bely M, et al. Adult hemangioma of the pancreas: difficult diagnosis of a rare disease. Clin J Gastroenterol. 2013;6(4):338-43.

2. Chang WT, Lee KT, Yang SF. Cavernous hemangioma of the pancreas: report of a case. Pancreas. 2003;26(3):310-2

3. Colardyn F, Elewaut A, Van de Velde E, et al. Hemangioma of the pancreas. Tijdschr Gastroenterol. 1972;15(4):260-7.

4. Dageforde J, Gmelin E, Otte M. Hemangioma of the pancreas. Rofo. 1991; 154(3):332-3.

5. Derom F, Ringoir S, Marlier R. Two cases of intraabdominal hemangioma: liver and pancreas. Acta Chir Belg. 1960;59:172-82.

6. Jarboui S, Salem A, Gherib BS, et al. Hemangioma of the pancreas in a 60year-old woman: a report of a new case. Gastroenterol Clin Biol. 2010;34(10): 569-71.

7. Kersting S, Janot MS, Munding J, et al. Rare solid tumors of the pancreas as differential diagnosis of pancreatic adenocarcinoma. JOP. 2012;13(3):268-77.

8. Kim SH, Kim JY, Choi JY, et al. Incidental detection of pancreatic hemangioma mimicking a metastatic tumor of renal cell carcinoma. Korean J Hepatobiliary Pancreat Surg. 2016;20(2):93-6.

9. Kobayashi $H$, Itoh T, Murata $R$, et al. Pancreatic cavernous hemangioma: CT, MRI, US, and angiography characteristics. Gastrointest Radiol. 1991;16(4):307-10.

10. Lee J, Raman K, Sachithanandan S. Pancreatic hemangioma mimicking a malignant pancreatic cyst. Gastrointest Endosc. 2011;73(1):174-6.

11. Lu T, Yang C. Rare case of adult pancreatic hemangioma and review of the literature. World J Gastroenterol. 2015:21(30):9228-32.

12. Lu ZH, Wu M. Unusual features in an adult pancreatic hemangioma: $\mathrm{CT}$ and MRI demonstration. Korean J Radiol. 2013;14(5):781-5.

13. Malik MAl, Kurban L. Pancreatic Hemangioma-a case report. Gastroenterol Hepatol Res. 2013:2:545-8.

14. Mangin P, Perret M, Ronjon A. Hemangioma of the pancreas. J Radiol. 1985; 66(5):381-4

15. Mondal U, Henkes N, Henkes D, et al. Cavernous hemangioma of adult pancreas: a case report and literature review. World J Gastroenterol. 2015; 21(33):9793-802

16. Naito $Y$, Nishida N, Nakamura $Y$, et al. Adult pancreatic hemangioma: a case report. Oncol Lett. 2014;8(2):642-4.

17. Plank CNB, Ba-Ssalamah A, Schima W. Pancreatic hemangioma: imaging features with contrast-enhanced CT and with gadolinium- and mangafodipir-enhanced MRI. Eur J Radiol. 2006;57:59-62.

18. Ringoir S, Derom F, Colle R, et al. Hemangioma of the pancreas. Report of a case. Gastroenterology. 1961;41:43-5.

19. Weidenfeld J, Zakai BB, Faermann $\mathrm{R}$, et al. Hemangioma of pancreas: a rare tumor of adulthood. Isr Med Assoc J. 2011;13(8):512-4.

20. Williamson JM, Finch-Jones M, Pope I. Endoscopic ultrasonography allowing expectant management of pancreatic haemangioma. Ann R Coll Surg Engl. 2014;96(3):e1-2

21. Xu Q, Wang $C F$, Zhao $P$, et al. The diagnosis and treatment of pancreatic cavernous hemangioma. Zhonghua Yi Xue Za Zhi. 2008;88(1):28-30.

22. Le Borgne J, de Calan L, Partensky C. Cystadenomas and cystadenocarcinomas of the pancreas: a multiinstitutional retrospective study of 398 cases. French Surgical Association. Ann Surg. 1999;230(2): 152-61. 
23. England RJ, Woodley H, Cullinane $\mathrm{C}$, et al. Pediatric pancreatic hemangioma a case report and literature review. JOP. 2006;7(5):496-501.

24. Hammel P, Beigelman C, Chauveau D, et al. Variety of pancreatic lesions observed in von Hippel-Lindau disease. Apropos of 8 cases. Gastroenterol Clin Biol. 1995;19(12):1011-7.

25. Mundinger GS, Gust S, Micchelli ST, et al. Adult pancreatic hemangioma: case report and literature review. Gastroenterol Res Pract. 2009;2009:839730.

26. Fang YF, Qiu LF, Du Y, et al. Small intestinal hemolymphangioma with bleeding: a case report. World J Gastroenterol. 2012;18(17):2145-6.

\section{Publisher's Note}

Springer Nature remains neutral with regard to jurisdictional claims in published maps and institutional affiliations.

Ready to submit your research? Choose BMC and benefit from:

- fast, convenient online submission

- thorough peer review by experienced researchers in your field

- rapid publication on acceptance

- support for research data, including large and complex data types

- gold Open Access which fosters wider collaboration and increased citations

- maximum visibility for your research: over $100 \mathrm{M}$ website views per year

At BMC, research is always in progress.

Learn more biomedcentral.com/submissions 